Highly Energetic Physical Processes and

Mechanisms for Emission from Astrophysical Plasmas

IAU Symposium, Vol. 195, 2000

P. C. H. Martens, S. Tsuruta, and M. A. Weber, eds.

\title{
Population Synthesis of GRB Progenitors: Problems With Kicks
}

\author{
C. L. Fryer \\ UCO/Lick Observatory, UC Santa Cruz, Santa Cruz, CA 95064, U.S.A.
}

\begin{abstract}
Accretion disks around stellar-mass black holes are now thought to be the engines which power classical gamma-ray bursts (GRBs). These disks are formed almost exclusively in binaries, and to study the characteristics of the progenitors of these black-hole accretion disk (BHAD) GRBs, we must understand the uncertainties in binary population synthesis calculations. Kicks imparted onto nascent neutron stars and black holes are among the most misunderstood concepts of binary population synthesis. In this paper, we outline the current understanding (or lack of understanding) of these kicks and discuss their effect on BHAD GRBs and binary population synthesis as a whole.
\end{abstract}

\section{Introduction}

The evidence for the cosmological origin of GRBs continues to grow, and with it has grown the popularity of a class of mechanisms driven by black holes accreting rapidly from a disk (see Popham, Woosley, \& Fryer 1999; Fryer, Woosley, \& Hartmann 2000 for reviews). These accretion disks are formed in a variety of ways: the merger of compact binaries including double neutron-star binaries (DNS) and binaries consisting of a black hole and either a neutron star or a white dwarf $(\mathrm{BH} / \mathrm{NS}, \mathrm{WD} / \mathrm{BH})$, the formation and subsequent accretion onto a black hole formed in the collapse of a massive star (collapsar), and the inspiral of a neutron star or black hole into a helium star companion (He-merger). In all of these formation scenarios, binary evolution plays a key role ${ }^{1}$. Binary population synthesis calculations can not only provide insight into the formation rates of these BHAD GRBs, but can also be used to determine redshift and spatial distributions of each of these progenitors. However, population synthesis is plagued by a variety of uncertainties. Here, we discuss one of the most misunderstood uncertainties (neutron-star and black-hole kicks) and the role it plays on BHAD GRB progenitors.

\section{Neutron Star Kicks}

The list of evidence for neutron-star kicks (velocities imparted to the neutron star at birth) continues to grow (see Fryer et al. 2000 for a review). The most

\footnotetext{
${ }^{1}$ Collapsars need not form in binaries, but in all our calculations, $99 \%$ form in binary scenarios.
} 
compelling evidence that many neutron stars receive large mean kick velocities $\left(\sim 450 \mathrm{~km} \mathrm{~s}^{-1}\right)$ comes from the observed distribution of pulsar velocities. Unfortunately, extracting the actual 3-dimensional kick from the observed proper motions of nearby pulsars suffers from a range of difficulties, from uncertainties in the effects of binaries and the Galactic potential to low number statistics and observational systematics and biases. The most recent attempts at determining the pulsar velocity distribution can be summarized as follows: Maxwellian distribution with mean velocity of $\sim 450,250-300 \mathrm{~km} \mathrm{~s}^{-1}$ (Lyne \& Lorimer 1994; Hansen \& Phinney 1997, respectively), and a bimodal distribution with a mean velocity of $\sim 450 \mathrm{~km} \mathrm{~s}^{-1}$ (Cordes \& Chernoff 1998). Fryer, Burrows, \& Benz (1998) went one step further and found that the neutron-star kick distribution is also bimodal with a mean velocity of $\sim 450 \mathrm{~km} \mathrm{~s}^{-1}$. For high kick velocities, the net effect of binaries on the mean pulsar velocity is minimal. However, binaries do spread the velocity distribution, so the neutron-star kick distribution may not have the same form as the observed pulsar velocity distribution.

The only discrepant result, that of Hansen \& Phinney (1997), is most probably due to incorrectly calculating the biases (Fryer et al. 1998). However, despite the amazing agreement in the estimates of neutron-star kicks, many papers using population synthesis codes persist in using much lower kick velocities, often citing Cordes \& Chernoff (1998) as their source. Cordes \& Chernoff (1998) characterize their velocities by standard deviations, and some readers have interpreted these standard deviations as the mean 3-dimensional velocities. In fact, they clearly state (p. 325) how to interpret their results and calculate a mean velocity of $479 \mathrm{~km} \mathrm{~s}^{-1}$ for a single Maxwellian distribution and a mean velocity of $421 \mathrm{~km} \mathrm{~s}^{-1}$ for a double peaked distribution. By doing very careful error analysis, Cordes \& Chernoff (1998) found that their bimodal distribution fits the observed pulsar distribution much better than a single Maxwellian. Fryer et al. (1998) came to the same conclusions, but by arguing that a high-velocity peak of kicks is required to explain the observed pulsars, while low velocities are required to explain $\mathrm{X}$-ray binaries.

The current data suggests that neutron stars receive kicks of over $400 \mathrm{~km} \mathrm{~s}^{-1}$. However, new studies on pulsar proper motions imply that many of the observed proper motions are incorrect by far more than their printed error bars. Systematic shifts of a factor of 2 in the observed pulsar velocity distribution are still possible (S. Thorsett, private comm.)! A decrease in the pulsar velocities by a factor of 2 would be welcome relief for theorists studying neutron-star kicks, who, at this point, do not have a mechanism which is able to produce the high observed pulsar velocities, when studied in detail. The current neutron-star kick mechanisms can be grouped into two basic categories: those mechanisms which produce kicks due to some asymmetry in the supernova explosion (either an asymmetry in the neutrino emission or in the ejecta), or those mechanisms which produce kicks due to asymmetries in the post-supernova fallback. These kick mechanisms have implications for black-hole kicks as well, and we will discuss them in more detail in $\S 3$.

\subsection{Population Synthesis and Kicks}

To illustrate the effects of kicks on binaries, let's return to our list of BHAD GRB progenitors. Using the code and standard parameter set described in 


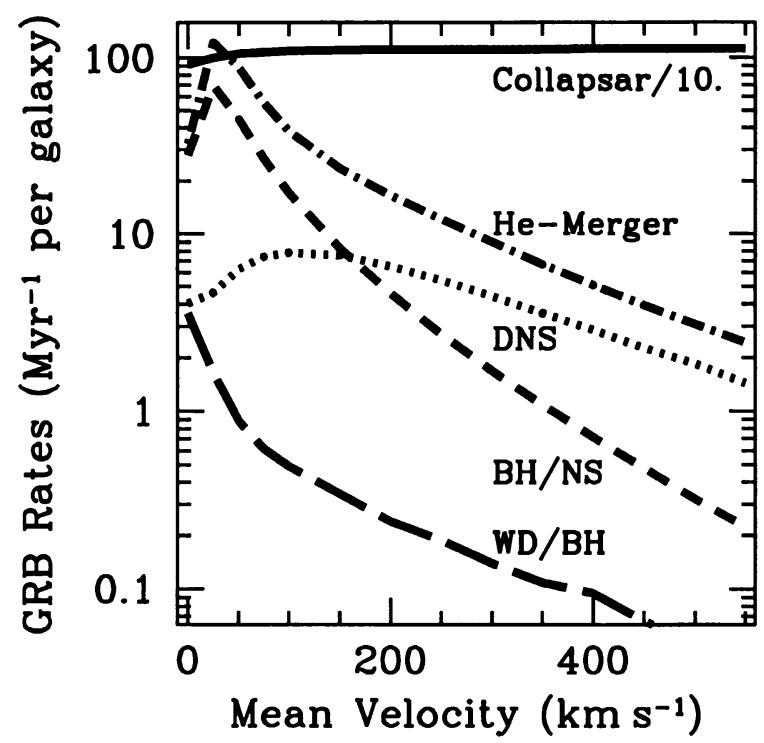

Figure 1. GRB rates for the 5 BHAD GRB progenitors vs. mean velocity assuming Maxwellian kick distributions. For the DNS, NS/BH, and He-merger progenitors, the rate initially increases with kick velocity. Most collapsars form in binaries (which leads to the ejection of the primary's hydrogen envelope) but are not affected by kicks.

Fryer et al. (2000), we have run a series of population synthesis calculations assuming Maxwellian kick distributions with a range of mean velocities. ${ }^{2}$ Note that the rates of DNS, $\mathrm{BH} / \mathrm{NS}$, and He-mergers all initially increase as the mean kick velocity rises from $0 \mathrm{~km} \mathrm{~s}^{-1}$ (Figure 1). In general, one would assume that neutron-star and black-hole kicks tend to unbind systems, producing fewer binaries. Indeed, as we increase the mean kick velocities, the number of binary systems does decrease. But the fraction of these binaries which actually merge is lower at lower kick velocities (Figure 2).

Why does this occur? It is well-known that kicks are necessary to explain the short-period DNS binaries in our Galaxy (see Fryer \& Kalogera 1997 or Wex, Kalogera, \& Kramer 2000 for the most recent work). The problem is the following: the formation scenarios of both DNS and BH/NS binaries pass through a phase where the primary star has collapsed to form a compact object which orbits around its companion (generally a helium star). To make a DNS or NS/BH binary with an orbital separation less than the radius of the helium

${ }^{2}$ I assume black holes and neutron stars receive kicks from the same Maxwellian distribution. For the most part, the rates of $\mathrm{DNS}, \mathrm{BH} / \mathrm{NS}$, and He-mergers depend on the neutron-star kicks. The rates of $\mathrm{BH} / \mathrm{WD}$ binaries depend only on the black-hole kick. 


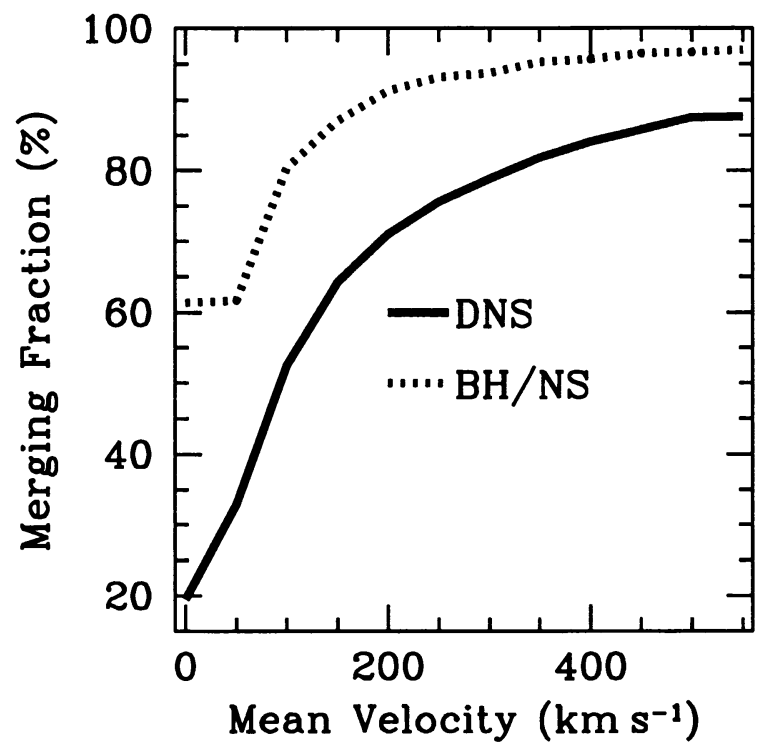

Figure 2. The fraction of DNS and NS/BH binaries which merge within a Hubble time vs. mean kick velocity. Kicks produce binaries with shorter periods!

star, one must use a kick during the collapse of the secondary to tighten the orbit.

Although high kick velocities are necessary to form close binaries, they unbind most wide binaries. This trend is easily seen in a series of eccentricityperiod distributions for different mean kick velocities (Figure 3). For the low kick velocities, binaries which never enter a common envelope remain bound (these are the population of wide orbit binaries between $~ 1-10$ A.U. which are present only in the low kick simulation). Note that the minimum orbital separation also decreases with increasing kick magnitude. The log-mean separations for DNS and $\mathrm{BH} / \mathrm{NS}$ binaries using a mean kick magnitude of $50 \mathrm{~km} \mathrm{~s}^{-1}$ are 16.0 and 41.5 $R_{\odot}$, respectively. For a mean kick velocity of $450 \mathrm{~km} \mathrm{~s}^{-1}$, these values decrease to 3.72 and $3.99 \mathrm{R}_{\odot}$.

\section{Black Hole Kicks}

The observational evidence for kicks seems to suggest that black-hole kicks are much weaker than neutron-star kicks. Most of this evidence is based on the scale height of the observed black-hole X-ray binaries (White \& van Paradijs 1996). Because black-hole X-ray binaries seem to be clustered close to the galactic plane, they argue that the mean systemic velocities of these binaries must be low $\left(42 \mathrm{~km} \mathrm{~s}^{-1}\right)$. However, we must remember that our observations are biased toward the galactic plane, and that our sample of black-hole $\mathrm{x}$-ray binaries has a much lower scale height than the true scale height of black-hole binaries. Indeed, two of the systems among the 10 objects in their sample have 

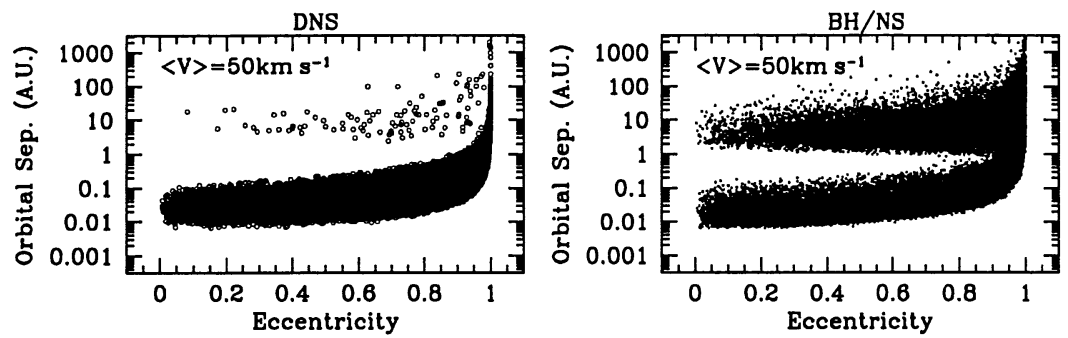

Figure 3. Distribution of orbital separations and eccentricity for DNS and $\mathrm{BH} / \mathrm{NS}$ binaries using a range of mean kick velocities. The population of wide-orbit binaries are systems which have avoided a common envelope phase, and which are disrupted when the kick magnitude is large. Note that the minimum orbital separation decreases with increasing kick magnitude.

velocities in excess of this velocity dispersion: Cyg X-1 and GRO J1655-40. In the case of GRO J1655-40, Bailyn et al. (1995) found that the radial velocity is $114 \pm 19 \mathrm{~km} \mathrm{~s}^{-1}$, which corresponds to a 3 -D velocity of $\sim 203 \mathrm{~km} \mathrm{~s}^{-1}$, nearly 5 sigma away from the White \& Paradijs (1996) result. With only 10 systems, we are either very lucky, or we have underestimated the black-hole X-ray binary velocity distribution.

Theories on kick mechanisms can predict an entire range of black-hole kicks. For example, if most black holes form through the direct collapse of a massive star, then many kick mechanisms driven by asymmetries in core-collapse supernova would not work, and these theories would predict no kicks for black holes. However, if these collapsars produce gamma-ray bursts and the GRB jets are slightly asymmetrical, then black holes could have jets which are much stronger than those of neutron stars (a $20 \%$ asymmetry in a $10^{53}$ erg burst produces a $1000 \mathrm{~km} \mathrm{~s}^{-1}$ kick). In population synthesis, however, we generally assume less extreme cases: either a) black holes receive kicks with mean velocities equal to neutron-star kicks, or b) black holes receive kicks with mean momenta equal to neutron-star kicks $\left(V_{\mathrm{BH}}=V_{\mathrm{NS}} M_{\mathrm{NS}} / M_{\mathrm{BH}}\right)$. Since it is likely that most black holes are formed in weak supernovae which have a lot of fallback, the kick mechanisms for neutron stars will produce black-hole kicks which fall into one of these two categories.

\subsection{Population Synthesis and Black Hole Kicks}

Our population synthesis calculations allow us to calculate systemic velocities of black-hole binaries with low-mass companions (Figure 4). These calculations include the effects of mass ejection (typically, the black-hole mass is one-third the mass of the initial star mass), but the dominant "kick" must be given by some asymmetry in the explosion that occured during black-hole formation (either the supernova or possibly a gamma-ray burst event). Many of these systems will become low-mass X-ray binaries. From Figure 4, we see that $100 \%, 99.5 \%$, $40 \%$ of black-hole binaries have velocities less than $100 \mathrm{~km} \mathrm{~s}^{-1}$, for mean kick 


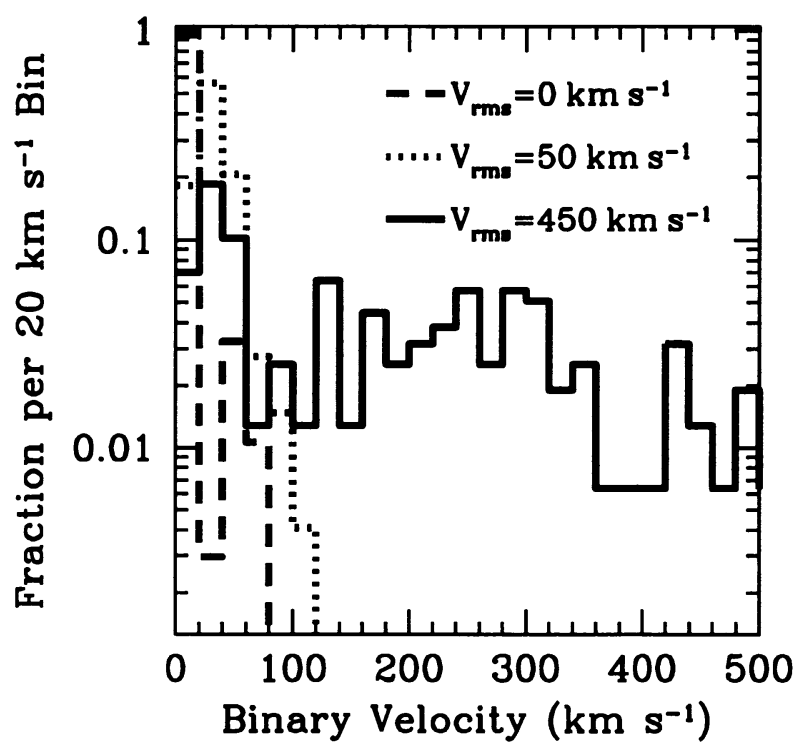

Figure 4. Black-hole binary velocity distribution for a range of blackhole kicks. The "no-kick" solution can not fit all of the data.

velocities of $0,50,450 \mathrm{~km} \mathrm{~s}^{-1}$, respectively. Clearly, the "no black-hole kick" solution is ruled out in the current data, primarily because this solution can not explain GRO J1655-40. However, one could argue that only in rare cases (10$20 \%$ ) do black holes receive kicks. Even then, one would have trouble explaining the $40 \mathrm{~km} \mathrm{~s}^{-1}$ mean inferred by White \& Paradijs (1996).The simulation using a mean velocity of $50 \mathrm{~km} \mathrm{~s}^{-1}$ seems to fit the data much better, although it too may have difficulty explaining GRO J1655-40. This mean kick velocity is roughly what one might expect from a kick mechanism which imparts equal momentum to neutron stars and black holes $\left(V_{\mathrm{BH}}=V_{\mathrm{NS}} M_{\mathrm{NS}} / M_{\mathrm{BH}}\right)$.

On the other hand, assuming a mean kick velocity of $450 \mathrm{~km} \mathrm{~s}^{-1}$ (blackhole kick velocities equal to neutron-star kick velocities) seems to produce far too many high-velocity black-hole binaries. But this plot is misleading. First of all, we are dealing with low number statistics in our sample, and it is difficult to determine if the data is biased toward low-velocity systems. Certainly, the highest velocity systems will move well into the halo of the galaxy before they become X-ray binaries. In addition, if the black-hole kick distribution is bimodal (e.g., see Fryer et al. 1998), the low-velocity peak will form the bulk of the bound black-hole binaries. The observed black-hole X-ray binaries will only give information about this lower black-hole kick.

Figure 5 shows the distribution of velocities for black-hole binaries versus black-hole mass and orbital separation. Note that the velocities are higher for low-mass black holes $\left(3-6 \mathrm{M}_{\odot}\right)$. This makes these objects more difficult to detect 

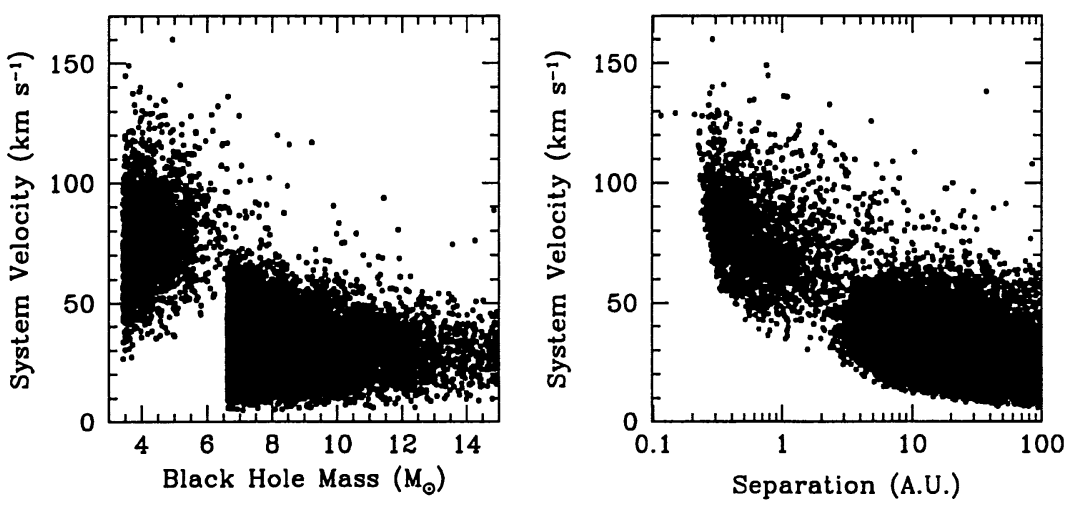

Figure 5. Distribution of systemic velocities for black-hole binaries using a Maxwellian kick distribution with mean velocity of $50 \mathrm{~km} \mathrm{~s}^{-1}$ vs. black-hole mass and orbital separation. The systemic velocities are higher for black holes in the range of $3-6 \mathrm{M}_{\odot}$, and hence these objects are more difficult to detect, a bias which must be understood to construct a black-hole mass distribution.

as they are more likely to move out of the plane of the galactic disk. This bias must be understood before we can construct a black-hole mass distribution. The high-velocity systems also tend to be short-period binaries.

So what can we say about black-hole and neutron-star kicks? Definitely some black holes receive kicks, otherwise we couldn't explain fast-moving systems such as GRO J1655-40. With the current data, low black-hole kick velocities seem to fit the data best, which either suggests that a kick mechanism should impart the same momentum to black holes and neutron stars, or it suggests that black holes, like neutron stars, have a bimodal kick distribution, and that the low-velocity peak is close to $50-100 \mathrm{~km} \mathrm{~s}^{-1}$. However, I would not be surprised if future measurements of pulsar and X-ray binaries yield lower pulsar velocities and higher X-ray binary velocities, requiring that black holes receive kicks with magnitudes equal to or greater than neutron-star kicks. Only time will tell!

Acknowledgments. This research has been supported by NASA (NAG58128), and the US DOE ASCI Program (W-7405-ENG-48). It is a pleasure to thank Thomas Janka and Jim Cordes for their insight and advice.

\section{References}

Bailyn, C. D., Orosz, J. A., McClintock, J. E., \& Remillard, R. A. 1995, Nature, 378,157

Cordes, J. M., \& Chernoff, D. F. 1998, ApJ, 505, 315 
Fryer, C. L., Burrows, A., \& Benz, W. 1998, ApJ, 496, 333

Fryer, C. L., \& Kalogera, V. 1997, ApJ, 489, 244

Fryer, C. L., Woosley, S. E., \& Hartmann, D. H. 2000, ApJ, in press

Hansen, B. M. S., \& Phinney, E. S. 1997, MNRAS, 291, 569

Lyne, A. G., \& Lorimer, D. R. 1994, Nature, 369, 127

Popham, R., Woosley, S. E., \& Fryer, C. L. 1999, ApJ, 518, 356

Wex, N., Kalogera, V., \& Kramer, M. 2000, ApJ, submitted

White, N. E., \& van Paradijs, J. 1996, ApJ, 473, L25 